\title{
ON THE SOLUTION OF INTEGRAL EQUATIONS WITH STRONGLY SINGULAR KERNELS*
}

\author{
BY \\ A. C. KAYA AND F. ERDOGAN \\ Lehigh University
}

\begin{abstract}
In this paper some useful formulas are developed to evaluate integrals having a singularity of the form $(t-x)^{-m}, m \geqslant 1$. Interpreting the integrals with strong singularities in the Hadamard sense, the results are used to obtain approximate solutions of singular integral equations. A mixed boundary value problem from the theory of elasticity is considered as an example. Particularly for integral equations where the kernel contains, in addition to the dominant term $(t-x)^{-m}$, terms which become unbounded at the end points, the present technique appears to be extremely effective to obtain rapidly converging numerical results.
\end{abstract}

1. Introduction. The mixed boundary value problems in physics and engineering may generally be expressed in terms of a "singular" integral equation of the form

$$
\int_{D} k(\mathbf{t}, \mathbf{x}) f(\mathbf{t}) d \mathbf{t}=g(\mathbf{x}), \quad \mathbf{x} \in D
$$

where $g$ is a known bounded function and the kernel $k$ is usually singular. The nature of the singularity of $k$ is dependent on the choice of the density function $f$ in formulating the problem. For example, in one-dimensional integral equations arising from potential theory, if $f(t)$ is selected to be a "flux," then $k$ has an ordinary Cauchy singularity $(t-x)^{-1}$. On the other hand, if $f$ is a potential, then $k$ has a strong singularity of the form $(t-x)^{-2}$. Particularly in two-dimensional integral equations, formulating the problem in terms of a potential rather than a flux-type quantity has certain advantages. Because of this it is worthwhile to develop effective techniques for evaluating singular integrals with strong singularities. In actual physical problems the density function $f$ is either bounded or may have integrable singularities on the boundary of $D$. Thus, in one-dimensional integral equations the integral on the left-hand side of Eq. (1) may be interpreted in Cauchy principal value sense for a Cauchy kernel, whereas it would be

\footnotetext{
${ }^{*}$ Received October 23, 1985.
} 
unbounded in the case of a strong singularity $(t-x)^{-2}$. Despite this, in the latter case the physical problem can still be solved provided the integral is interpreted in the Hadamard sense by retaining the finite part only.

The concept of finite-part integrals was first introduced by Hadamard [1] in connection with divergent integrals of the form

$$
\int_{a}^{x} \frac{f(t)}{(x-t)^{p+\frac{1}{2}}} d t
$$

where $f$ is bounded and $p$ is an integer. In spite of this relatively early beginning, the adoption of the concept in applications has been rather slow $[2,3]$. It is mainly due to Kutt's work [4-6] that the idea is finding relatively wide applications. To demonstrate Hadamard's basic idea we consider the integral

$$
S_{0}(x)=\int_{x}^{b} \frac{d t}{(t-x)^{1 / 2}}=2(b-x)^{1 / 2} \quad(x<b),
$$

from which, differentiating both sides separately, it follows that

$$
\frac{d}{d x} S_{0}(x)=\frac{1}{2} \int_{x}^{b} \frac{d t}{(t-x)^{3 / 2}}-\left.\frac{1}{(t-x)^{1 / 2}}\right|_{t=x}=-\frac{1}{(b-x)^{1 / 2}} \quad(x<b) .
$$

In (4) it is seen that the derivative of $S_{0}$ (which is bounded) is the difference between a divergent integral and an unbounded integrated term. Noting that the integrated term is independent of $b$, we may now consider the derivative of $S_{0}$ as being the "finite part" of the divergent integral and define

$$
f_{x}^{b} \frac{d t}{(t-x)^{3 / 2}}=\lim _{c \rightarrow x}\left[\int_{c}^{b} \frac{d t}{(t-x)^{3 / 2}}-\frac{2}{(c-x)^{1 / 2}}\right]=-\frac{2}{(b-x)^{1 / 2}} \quad(x<c<b) \text {. }
$$

Following are some other examples (see also [5]):

$$
\begin{aligned}
f_{x}^{b} \frac{d t}{(t-x)^{\alpha+1}} & =\lim _{c \rightarrow x}\left[\int_{c}^{b} \frac{d t}{(t-x)^{\alpha+1}}-\frac{1}{\alpha} \frac{1}{(c-x)^{\alpha}}\right]=-\frac{1}{\alpha}(b-x)^{-\alpha} \quad(\alpha>0), \\
f_{x}^{b} \frac{d t}{t-x} & =\lim _{c \rightarrow x}\left[\int_{c}^{b} \frac{d t}{t-x}+\log (c-x)\right]=\log (b-x) \quad(x<b), \\
f_{a}^{b} \frac{d t}{t-x} & =f_{a}^{x} \frac{d t}{t-x}+f_{x}^{b} \frac{d t}{t-x}=\log (b-x)-\log (x-a) \quad(a<x<b), \\
f_{a}^{b} \frac{d t}{(t-x)^{2}} & =f_{a}^{x} \frac{d t}{(t-x)^{2}}+f_{x}^{b} \frac{d t}{(t-x)^{2}}=-\frac{1}{b-x}-\frac{1}{x-a} \quad(a<x<b),
\end{aligned}
$$


$\frac{d}{d x} \int_{a}^{b} f(t) \log |t-x| d t=-f_{a}^{b} \frac{f(t)}{t-x} d t \quad(a<x<b)$,

$\frac{d}{d x} f_{a}^{b} \frac{f(t)}{t-x} d t=f_{a}^{b} \frac{f(t)}{(t-x)^{2}} d t \quad(a<x<b)$

$\frac{d}{d x} f_{a}^{b} \frac{f(t)}{(t-x)^{\alpha+1}} d t=f_{a}^{b} \frac{\partial}{\partial x}\left[\frac{1}{(t-x)^{\alpha+1}}\right] f(t) d t \quad(a<x<b, \alpha>0)$.

In this paper some useful formulas for the evaluation of certain singular integrals are developed. The results are then used to obtain effective numerical solutions to integral equations having kernels with strong singularities and some examples are given.

2. Evaluation of finite-part integrals. With an eye on applications to one-dimensional mixed boundary value problems, in this section we will describe some simple techniques for evaluating the finite-part integrals having $(t-x)^{-2}$ as the kernel. Let $F(t)$ be a bounded function with continuous first and second derivatives and let the interval be normalized such that $-1<x, t<1$. The singular integral may then be expressed as

$$
\begin{aligned}
f_{-1}^{1} \frac{F(t) w(t)}{(t-x)^{2}} d t= & \int_{-1}^{1}\left[F(t)-F(x)-(t-x) F^{\prime}(x)\right] \frac{w(t)}{(t-x)^{2}} d t \\
& +F(x) f_{-1}^{1} \frac{w(t) d t}{(t-x)^{2}}+F^{\prime}(x) f_{-1}^{1} \frac{w(t) d t}{t-x} \quad(-1<x<1),
\end{aligned}
$$

where $w(t)$ is the fundamental function of the corresponding mixed boundary value problem and may be determined by using a suitable function-theoretic method [7]. For simple physical problems $w$ is given by one of

$$
w(t)=1, \quad\left(1-t^{2}\right)^{\mp 1 / 2}, \quad(1-t)^{\mp 1 / 2} .
$$

One may now note that the first integral on the right-hand side of Eq. (13) is bounded (the integrand approaches $\frac{1}{2} F^{\prime \prime}(x) w(x)$ as $\left.t \rightarrow x\right)$ and the remaining integrals may be evaluated by using the following expressions:

$$
\begin{aligned}
f_{-1}^{1} \frac{d t}{t-x} & =\log \left|\frac{1-x}{1+x}\right| \\
f_{-1}^{1} \frac{d t}{(t-x)^{2}} & =-\frac{1}{1-x}-\frac{1}{1+x}, \\
f_{-1}^{1} \frac{\sqrt{1-t^{2}}}{t-x} d t & =-\pi x \quad(-1<x<1), \\
f_{-1}^{1} \frac{\sqrt{1-t^{2}}}{(t-x)^{2}} d t & =-\pi \quad(-1<x<1),
\end{aligned}
$$




$$
\begin{gathered}
f_{-1}^{1} \frac{d t}{(t-x) \sqrt{1-t^{2}}}=0 \quad(-1<x<1) \\
f_{-1}^{1} \frac{d t}{(t-x)^{2} \sqrt{1-t^{2}}}=0 \quad(-1<x<1) \\
f_{-1}^{1} \frac{\sqrt{1-t}}{t-x} d t=\sqrt{1-x} \log |B|-2 \sqrt{2} \quad(x<1) \\
f_{-1}^{1} \frac{\sqrt{1-t}}{(t-x)^{2}} d t=-\frac{\log |B|}{2 \sqrt{1-x}}-\frac{\sqrt{2}}{1+x} \quad(x<1) \\
f_{-1}^{1} \frac{d t}{(t-x) \sqrt{1-t}}=\frac{\log |B|}{\sqrt{1-x}} \quad(x<1) \\
f_{-1}^{1} \frac{d t}{(t-x)^{2} \sqrt{1-t}}=\frac{1}{1-x}\left(\frac{\log |B|}{2 \sqrt{1-x}}-\frac{\sqrt{2}}{1+x}\right) \quad(x<1) \\
f_{-1}^{1} \frac{\sqrt{1-t}}{(t-x)^{m}} d t=-\frac{2 \sqrt{2}(-1)^{m}}{(m-1)(1-x)(1+x)^{m-1}} \\
\quad+\frac{2 m-5}{2(m-1)(1-x)} f_{-1}^{1} \frac{\sqrt{1-t}}{(t-x)^{m-1}} d t \quad(x<1) \quad(25) \\
f_{-1}^{1} \frac{d t}{(t-x)^{m} \sqrt{1-t}}=-\frac{2 m-3}{(m-1)(1-x)(1+x)^{m-1}} f_{-1}^{1} \frac{d t}{(t-x)^{m-1} \sqrt{1-t}} \\
\quad(m-1)(1-x)
\end{gathered}
$$

where $m$ is an integer $(m \geqslant 2)$ and

$$
B=\left(1+\sqrt{\frac{1-x}{2}}\right) /\left(1-\sqrt{\frac{1-x}{2}}\right) .
$$

The expressions involving the weight functions $\left(1-t^{2}\right)^{\mp 1 / 2}$ are standard and may be found in handbooks or derived directly by using the complex function theory [7]. Formulas (21) and (23) are obtained by using certain properties of Jacobi polynomials (see Appendix A); the remaining formulas given above may then be obtained from (21) and (23) by differentiation (see (11) and (12)).

In solving integral equations it is often convenient to express the unknown function $F(t)$ in terms of a polynomial with undetermined coefficients. In such problems the following expressions may be quite useful:

$$
\begin{aligned}
f_{-1}^{1} \frac{P_{n}(t)}{t-x} d t & =-2 Q_{n}(x), \\
f_{-1}^{1} \frac{P_{n}(t)}{(t-x)^{2}} d t & =-\frac{2(n+1)}{1-x^{2}}\left[x Q_{n}(x)-Q_{n+1}(x)\right],
\end{aligned}
$$




$$
\begin{aligned}
f_{-1}^{1} \frac{U_{n}(t) \sqrt{1-t^{2}}}{t-x} d t & =-\pi T_{n+1}(x) \quad(n \geqslant 0) \\
f_{-1}^{1} \frac{U_{n}(t) \sqrt{1-t^{2}}}{(t-x)^{2}} d t & =-\pi(n+1) U_{n}(x) \quad(n \geqslant 0) \\
f_{-1}^{1} \frac{T_{n}(t)}{(t-x) \sqrt{1-t^{2}}} d t & = \begin{cases}0 & (n=0) \\
\pi U_{n-1}(x) & (n \geqslant 1)\end{cases} \\
f_{-1}^{1} \frac{T_{n}(t)}{(t-x)^{2} \sqrt{1-t^{2}}} d t & = \begin{cases}0 \quad(n=0,1) \\
\frac{\pi}{1-x^{2}}\left[-\frac{n-1}{2} U_{n}(x)+\frac{n+1}{2} U_{n-2}(x)\right]\end{cases}
\end{aligned}
$$

where $T_{n}$ and $U_{n}$ are Chebyshev polynomials of the first and second kind, respectively, $P_{n}$ is the Legendre polynomial, and $Q_{n}$ is the Legendre function of the second kind. Also, referring to Appendix B it can be shown that

$$
\begin{aligned}
& B_{n}(x)=\frac{1}{\pi} f_{-1}^{1} \frac{t^{n} \sqrt{1-t^{2}}}{t-x} d t=\sum_{k=0}^{n+1} b_{k} x^{k} \quad(n \geqslant 0), \\
& C_{n}(x)=\frac{1}{\pi} f_{-1}^{1} \frac{t^{n} \sqrt{1-t^{2}}}{(t-x)^{2}} d t=\sum_{k=0}^{n} c_{k} x^{k} \quad(n \geqslant 0) \\
& D_{n}(x)=\frac{1}{\pi} f_{-1}^{1} \frac{t^{n} d t}{(t-x) \sqrt{1-t^{2}}}=\left\{\begin{array}{l}
0 \quad(n=0) \\
\sum_{k=0}^{n-1} d_{k} x^{k} \quad(n \geqslant 1),
\end{array}\right. \\
& E_{n}(x)=\frac{1}{\pi} f_{-1}^{1} \frac{t^{n} d t}{(t-x)^{2} \sqrt{1-t^{2}}}=\left\{\begin{array}{l}
0 \quad(n=0,1) \\
\sum_{k=0}^{n-2} e_{k} x^{k} \quad(n \geqslant 2),
\end{array}\right. \\
& R_{n}^{j}(x)=f_{-1}^{1} \frac{t^{n} \sqrt{1-t}}{(t-x)^{j}} d t=\sum_{m=1}^{j}\left(\begin{array}{c}
n \\
j-m
\end{array}\right) x^{n-j+m} f_{-1}^{1} \frac{\sqrt{1-t}}{(t-x)^{m}} d t \\
& +\sum_{k=0}^{n-j} A_{k}^{j} x^{n-j-k} \quad(x<1, n \geqslant 0) \\
& =\sum_{m=1}^{j}\left(\begin{array}{c}
n \\
j-m
\end{array}\right) x^{n-j+m} f_{-1}^{1} \frac{d t}{(t-x)^{m} \sqrt{1-t}}+\sum_{k=0}^{n-j} B_{k}^{j} x^{n-j-k} \quad(x<1, n \geqslant 0),
\end{aligned}
$$


where the coefficients $b_{k}, c_{k}, d_{k}, e_{k}, A_{k}^{j}$, and $B_{k}^{j}$ are given in Appendix B and $\left(\begin{array}{c}n \\ i\end{array}\right)$ is the binomial coefficient. Appendix B also shows the derivation of $R_{n}^{j}(x)$. A similar technique was used to obtain the functions $B_{n}, C_{n}, D_{n}, E_{n}$, and $S_{n}^{j}$. The appendix also gives some useful recursion formulas involving the functions defined by (34-39).

Even though there are also Gaussian-type integration formulas developed by Kutt [5] for the evaluation of the singular integral

$$
f_{x}^{b} \frac{f(t)}{(t-x)^{\lambda}} d t, \quad \lambda \geqslant 1
$$

they are not very convenient for solving integral equations by using the standard quadrature method which requires the use of fixed stations $t_{i}$, since in Kutt's formulas the $t_{i}$ vary as $x$ is changed (see also [8]).

3. Solution of integral equations. Let us now assume that the mixed boundary value problem is reduced to the following one-dimensional integral equation:

$$
\int_{a}^{b}\left[k_{s}(t, x)+k(t, x)\right] f(t) d t=g(x) \quad(a<x<b),
$$

where the kernel $k$ is square integrable in $[a, b]$ and $g$ is a known bounded function. If the unknown function $f$ is a "potential" quantity, then the singular kernel $k_{s}$ has a strong singularity (i.e., it contains terms of the order $(t-x)^{-n}, n>1$ ). The fundamental (or the weight) function $w(t)$ of the problem may be determined from $k_{s}$, and $f$ may be expressed as

$$
f(t)=F(t) w(t), \quad a<t<b,
$$

where $F$ is an unknown bounded function. In solving the integral equations with strong singularities, the application of standard quadrature formulas does not seem to be very practical. In these problems the simplest and the most effective technique appears to be to approximate the unknown function $F$ by a truncated series as

$$
F(t) \cong \sum_{n=0}^{N} a_{n} \phi_{n}(t)
$$

and to determine the coefficients $a_{n}$ by a weighted residual method. Here $\left\{\phi_{n}\right\}$ may be any convenient complete system of functions. Substituting from (42) and (43) into (41) we obtain

$$
\sum_{n=0}^{N} a_{n} G_{n}(x) \cong g(x) \quad(a<x<b)
$$

where

$$
G_{n}(x)=f_{a}^{b} k_{s}(t, x) \phi_{n}(t) w(t) d t+\int_{a}^{b} k(t, x) \phi_{n}(t) w(t) d t .
$$

The coefficients $a_{n}$ may then be determined from the following system of algebraic equations:

$$
\sum_{n=0}^{N} a_{n} \int_{a}^{b} G_{n}(x) \psi_{j}(x) w_{j}(x) d x=\int_{a}^{b} g(x) \psi_{j}(x) w_{j}(x) d x \quad(j=0,1, \ldots, N),
$$


where $\psi_{j}$ is a coordinate function in a complete system (e.g., a set of orthonormal polynomials) and $w_{j}$ is the corresponding weight. The functions $\phi_{n}(t)$ and $\psi_{j}(x)$ are usually selected in such a way that their orthogonality properties may be utilized. In practice one may use trigonometric functions, Legendre polynomials, Chebyshev polynomials, delta functions, or any linearly independent set of polynomials such as $t^{n}$ and $x^{j}$. Quite clearly the numerical work in (46) may be reduced considerably if we select

$$
w_{j}(x)=1, \quad \psi_{j}(x)=\delta\left(x-x_{j}\right) \quad(j=0,1, \ldots, N) .
$$

By doing so we can use a simple collocation method to reduce (44) to the following algebraic system:

$$
\sum_{n=0}^{N} a_{n} G_{n}\left(x_{j}\right)=g\left(x_{j}\right) \quad(j=0,1, \ldots, N)
$$

Although the collocation points $x_{j}$ can be selected arbitrarily, in general they are chosen as the roots of Legendre or Chebyshev polynomials. Even though there is no restriction on the choice of $x_{j}$, our experience shows that a symmetric distribution with respect to the origin with more points concentrated near the ends seems to help (see also [9]). One may also note that in the case of a resulting ill-conditioned system, one could select $(M+1)$ coordinate functions $\psi_{j}$ with $M>N$ in (46) or (47) and determine $(N+1)$ unknowns $a_{n}$ from a set of $(M+1)$ equations by using the method of least squares.

Needless to say, if the integral equation (41) contains only a dominant kernel $(t-x)^{-1}$ or $(t-x)^{-2}$, one may always obtain the closed form solution by expanding the functions $g(x)$ and $F(t)$ into appropriate series and by using the results given in the previous section and Appendix B.

4. Applications: A crack in an infinite strip. In fracture mechanics the problem of an infinite strip containing a crack perpendicular to its boundaries has been of wide interest, since this geometry can be used as an approximation to a number of structural components and laboratory specimens. The related boundary value problem will be discussed below and the numerical treatment of the resulting singular integral equation will be given to demonstrate the solution technique that was outlined in the previous section.

As shown in Fig. 1, the crack lies perpendicular to the stress-free boundaries and is under prescribed surface tractions $p(x)$. In terms of the crack opening displacement defined by

$$
V(x)=u_{y}(x,+0)-u_{y}(x,-0) \quad(a<x<b),
$$

the problem may be reduced to the following integral equation (see [10] and Appendix C).

$$
f_{a}^{b} \frac{V(t)}{(t-x)^{2}} d t+\int_{a}^{b} V(t) K(t, x) d t=-\pi\left(\frac{1+\kappa}{2 \mu}\right) p(x), a<x<b,
$$


where the kernel $K(t, x)$ is given by

$$
\begin{aligned}
K(t, x)= & K_{1}(t, x)+K_{1}(h-t, h-x)+K_{2}(t, x)+K_{2}(h-t, h-x), \\
K_{1}(t, x)= & -\frac{1}{(t+x)^{2}}+\frac{12 x}{(t+x)^{3}}-\frac{12 x^{2}}{(t+x)^{4}}, \\
K_{2}(t, x)= & \int_{0}^{\infty}\left[f_{1}(t, x, \alpha) e^{-\alpha(t+x)}+f_{2}(t, x, \alpha) e^{-\alpha(2 h+x-t)}\right] d \alpha, \\
f_{1}(t, x, \alpha)= & \frac{\alpha}{D} e^{-2 \alpha h}\left\{8 \alpha^{4} h^{2} t x-12 \alpha^{3} h^{2}(t+x)+2 \alpha^{2}\left[9 h^{2}+h(t+x)+t x\right]\right. \\
& \left.-3 \alpha[2 h+t+x]+5+e^{-2 \alpha h}\left[-2 \alpha^{2} t x+3 \alpha(t+x)-5\right]\right\}, \\
f_{2}(t, x, \alpha)= & \frac{\alpha}{D}\left\{-4 \alpha^{3}[h x(h-t)]+6 \alpha^{2}\left[h^{2}+h(x-t)\right]\right. \\
& \left.+\alpha[-10 h+t-x]+3+e^{-2 \alpha h}[\alpha(x-t)-3]\right\}, \\
D= & 1-\left(4 \alpha^{2} h^{2}+2\right) e^{-2 \alpha h}+e^{-4 \alpha h} .
\end{aligned}
$$

Note that for $h \rightarrow \infty, K_{1}(h-t, h-x)$ and $K_{2}$ vanish and the integral equation for the half-plane is recovered.

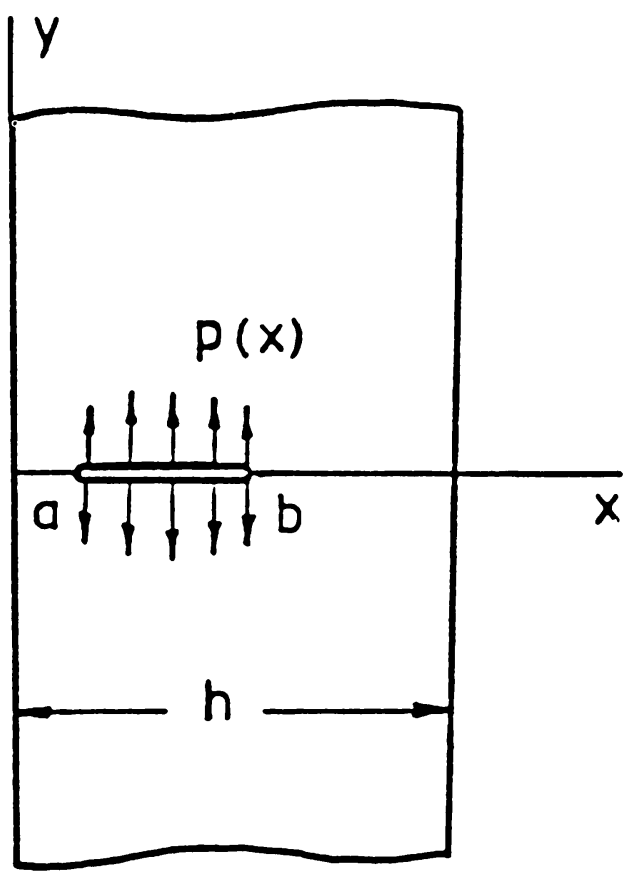

FIG. 1. A Crack in an Infinite Strip 
Normalizing the interval $(a, b)$ by defining

$$
\begin{gathered}
t=\left(\frac{b-a}{2}\right) r+\left(\frac{b+a}{2}\right), \quad x=\left(\frac{b-a}{2}\right) s+\left(\frac{b+a}{2}\right), \\
V(t)=\left(\frac{b-a}{2}\right) f(r)
\end{gathered}
$$

the integral equation (50) becomes

$$
f_{a}^{b} \frac{f(r)}{(r-s)^{2}} d r+\int_{-1}^{1} f(r) k(r, s) d r=g(x), \quad-1<s<1,
$$

where

$$
k(r, s)=\left(\frac{b-a}{2}\right)^{2} K(t, x), \quad g(x)=-\pi\left(\frac{1+\kappa}{2 \mu}\right) p(x) .
$$

The cases $a>0$ and $a=0$ represent the internal and the edge crack, respectively, and these two problems will be treated separately. In each case the solution will be assumed to be of the form

$$
f(r)=F(r) w(r)
$$

where the fundamental solution $w(r)$ can be determined from the dominant behavior of the singular kernels in the integral equation and is found to be

$$
\begin{aligned}
& w(r)=\sqrt{1-r^{2}}, \quad \text { internal crack, } \\
& w(r)=\sqrt{1-r}, \quad \text { edge crack. }
\end{aligned}
$$

Internal crack: $a>0$. Following the procedure described in the previous section, $F(r)$ is now approximated in terms of a truncated series of Chebyshev polynomials,

$$
F(r)=\sum_{n=0}^{N} a_{n} U_{n}(r)
$$

By substituting from (59), (60), and (62) into (57) and by using (31), we obtain

$$
\sum_{n=0}^{N} a_{n}\left[-\pi(n+1) U_{n}(s)+h_{n}(s)\right]=g(s), \quad-1<s<1,
$$

where

$$
h_{n}(s)=\int_{-1}^{1} U_{n}(r) k(r, s) \sqrt{1-r^{2}} d r .
$$

The unknown coefficients $a_{n}$ are then determined from Eq. (63) by selecting a convenient set of collocation points such as the zeros of $T_{N+1}$,

$$
s_{j}=\cos \left(\frac{2 j+1}{N+1} \frac{\pi}{2}\right) \quad(j=0,1, \ldots, N) .
$$

Once the solution is obtained, the stress intensity factors, which are the main parameters of interest in fracture problems, can be calculated from

$$
\begin{array}{rlrl}
k_{1}(a) & =\lim _{x \rightarrow a} \sqrt{2(a-x)} \sigma_{y y}(x, 0), & & (x<a) \\
& =\left(\frac{2 \mu}{\kappa+1}\right) \lim _{t \rightarrow a} \frac{V(t)}{\sqrt{2(t-a)}}, \quad & & (t>a) \\
& =\left(\frac{2 \mu}{\kappa+1}\right) \sqrt{\frac{b-a}{2}} F(-1), &
\end{array}
$$




$$
\begin{array}{rlrl}
k_{1}(b) & =\lim _{x \rightarrow b} \sqrt{2(x-b)} \sigma_{y y}(x, 0), & & (x>b) \\
& =\left(\frac{2 \mu}{\kappa+1}\right) \lim _{t \rightarrow b} \frac{V(t)}{\sqrt{2(b-t)}}, \quad & & (t<b) \\
& =\left(\frac{2 \mu}{\kappa+1}\right) \sqrt{\frac{b-a}{2}} F(1) .
\end{array}
$$

Equations (66) and (67) are obtained from (50) by observing that the left-hand side in (50) gives the stress component $\sigma_{y y}(x, 0)$ outside as well as inside the cut $(a, b)[10]$.

Table 1 shows the stress intensity factors for an internal crack in a half-plane under uniform loading $p(x)=p_{0}$ as an example.

Edge crack: $a=0$. The solution of the integral equation (50) for $a=0$ needs more care. This is due to the fact that the kernel $K(t, x)$ becomes singular as $t$ and $x$ approach 0 simultaneously. (Similarly, in (57) $k(r, s)$ becomes unbounded as $r$ and $s$ approach -1.)

For a weight function $\sqrt{1-r}$, certain relations involving singular integrals of power series have been presented in Sec. 2. Therefore, if we express the unknown function $F(r)$ as

$$
F(r)=\sum_{n=0}^{N} a_{n} r^{n}
$$

the singular integrals may be evaluated from Eq. (38) by letting $j=2$. The integral equation (57) now becomes

where

$$
\sum_{n=0}^{N} a_{n} G_{n}(s)=g(s), \quad-1<s<1,
$$

$$
G_{n}(s)=f_{-1}^{1} \frac{r^{n} \sqrt{1-r}}{(r-s)^{2}} d r+\int_{-1}^{1} r^{n} \sqrt{1-r} k(r, s) d r
$$

TABLE 1. Normalized stress intensity factors for an internal crack in a half-plane. $(N+1)$ terms are used in approximating the unknown function.

\begin{tabular}{|c|c|c|c|}
$\left(\frac{b+a}{b-a}\right)$ & $\frac{k_{1}(a)}{p_{0} \sqrt{(b-a) / 2}}$ & $\frac{k_{1}(b)}{p_{0} \sqrt{(b-a) / 2}}$ & $N+1$ \\
\hline 1.01 & 3.6387 & 1.3298 & 15 \\
1.05 & 2.1547 & 1.2536 & 10 \\
1.1 & 1.7587 & 1.2108 & 10 \\
1.2 & 1.4637 & 1.1626 & 6 \\
1.3 & 1.3316 & 1.1331 & 6 \\
1.4 & 1.2544 & 1.1123 & 6 \\
1.5 & 1.2035 & 1.0967 & 4 \\
2.0 & 1.0913 & 1.0539 & 4 \\
3.0 & 1.0345 & 1.0246 & 4 \\
4.0 & 1.0182 & 1.0141 & 4 \\
5.0 & 1.0112 & 1.0092 & 4 \\
10.0 & 1.0026 & 1.0024 & 4 \\
20.0 & 1.0006 & 1.0006 & 4 \\
\hline
\end{tabular}


or using the notation of Eq. (38),

$$
G_{n}(s)=R_{n}^{2}(s)+\int_{-1}^{1} r^{n} \sqrt{1-r} k(r, s) d r .
$$

The integral in Eq. (71) can be evaluated numerically; however, as $s \rightarrow-1$, the value of the integral becomes unbounded. It may be observed that for $s=-1, R_{n}^{2}(s)$ is also unbounded, resulting in a bounded value for $G_{n}(-1)$. To determine the coefficients $a_{n}$, the collocation points may be selected as in Eq. (65).

For $h \rightarrow \infty$ the kernel $k(r, s)$ simply becomes

$$
k(r, s)=-\frac{1}{(r+s+2)^{2}}+\frac{12(s+1)}{(r+s+2)^{3}}-\frac{12(s+1)^{2}}{(r+s+2)^{4}},
$$

and from (71), (72), and (38) it can be shown that

$$
G_{n}(s)=R_{n}^{2}(s)-R_{n}^{2}(-s-2)+12(s+1) R_{n}^{3}(-s-2)-12(s+1)^{2} R_{n}^{4}(-s-2) .
$$

In the limiting case we find

$$
G_{n}(-1)=-\sqrt{2}(4 n+1)(-1)^{n}
$$

The stress inequality factor is given by

$$
\begin{array}{rlrl}
k_{1}(b) & =\lim _{x \rightarrow b} \sqrt{2(x-b)} \sigma_{y y}(x, 0) & (x>b) \\
& =\left(\frac{2 \mu}{\kappa+1}\right) \lim _{t \rightarrow b} \frac{V(t)}{\sqrt{2(b-t)}} \quad(t<b) \\
& =\left(\frac{2 \mu}{\kappa+1}\right) \frac{\sqrt{b}}{2} F(1) .
\end{array}
$$

As a first example we again consider a semi-infinite plane with an edge crack. In this case the kernel of the integral equation is given in closed form (see (57) and (72)) and the numerical analysis can be carried out quite accurately. For a uniform crack surface pressure $p(x)=p_{0}$ and for various values of $N$, the calculated stress intensity factor $k_{1}(b)$ and the relative crack opening displacement $V(0)$ are given in Table 2 . The table also shows the correct value of $k_{1}(b)$, which was calculated from the infinite integral given in [11] (see Appendix D). It is seen that the convergence of the method is extremely good.

The second example is concerned with a long strip of finite width $h$ which contains an edge crack of length $b$ and is subjected to a uniform tension $p_{0}\left(p(x)=p_{0}\right)$ or pure bending $M\left\{p(x)=\left(6 M / h^{2}\right)[1-2 x / h]\right\}$ away from the crack region. In the numerical analysis the number of terms in the series was increased until the accuracy of the last significant digits given in Table 3 was verified. In no case were more than 20 terms needed.

$\left(^{*}\right)$ Although (73) is valid for all $s<1$, due to high powers of $(-s-2)$ needed for large $n$, it should only be used near $s=-1$ to maintain good accuracy. 
TABLE 2. Normalized stress intensity factor and crack opening displacement for an edge crack in a half-plane.

\begin{tabular}{c|c|c}
$N+1$ & $\frac{k_{1}(b)}{p_{0} \sqrt{b}}$ & $\left(\frac{2 \mu}{1+\kappa}\right) \frac{V(0)}{p_{0} h}$ \\
\hline 1 & 1.062652 & 1.502816 \\
2 & 1.126950 & 1.423476 \\
3 & 1.124283 & 1.457747 \\
4 & 1.121818 & 1.455918 \\
5 & 1.121442 & 1.454520 \\
6 & 1.121451 & 1.454224 \\
7 & 1.121483 & 1.454211 \\
8 & 1.121504 & 1.454241 \\
9 & 1.121514 & 1.454264 \\
10 & 1.121518 & 1.454278 \\
15 & 1.121522 & 1.454298 \\
20 & 1.121522 & 1.454298 \\
\hline
\end{tabular}

${ }^{*}$ The correct value of stress intensity factor (calculated from the infinite integral given by Koiter [11]).

TABLE 3. Normalized stress intensity factor and the relative crack opening displacement for an edge crack in a long strip under uniform tension $p_{0}$ or pure bending $M$ away from the crack region;

$\sigma_{m}=6 M / h^{2}$

\begin{tabular}{|l|l|c|c|c|}
\hline \multirow{2}{*}{$b / h$} & \multicolumn{2}{|c|}{ Tension } & \multicolumn{2}{c|}{ Bending } \\
\cline { 2 - 5 } & $\frac{k_{1}(b)}{p_{0} \sqrt{b}}$ & $\left(\frac{2 \mu}{1+\kappa}\right) \frac{V(0)}{p_{0} h}$ & $\frac{k_{1}(b)}{\sigma_{m} \sqrt{b}}$ & $\left(\frac{2 \mu}{1+\kappa}\right) \frac{V(0)}{\sigma_{m} h}$ \\
\hline$\rightarrow 0$ & 1.12152226 & & 1.12152226 & \\
0.00001 & 1.121522 & $0.14543 \times 10^{-4}$ & 1.1215 & $0.14543 \times 10^{-4}$ \\
0.001 & 1.121531 & $0.14543 \times 10^{-2}$ & 1.1202 & $0.14535 \times 10^{-2}$ \\
0.1 & 1.1892 & 0.15490 & 1.0472 & 0.14529 \\
0.2 & 1.3673 & 0.36543 & 1.0553 & 0.31822 \\
0.3 & 1.6599 & 0.70358 & 1.1241 & 0.56141 \\
0.4 & 2.1114 & 1.3048 & 1.2606 & 0.94130 \\
0.5 & 2.8246 & 2.4702 & 1.4972 & 1.5924 \\
0.6 & 4.0332 & 4.9746 & 1.9140 & 2.8387 \\
0.7 & 6.3549 & 11.246 & 2.7252 & 5.6432 \\
0.8 & 11.955 & 31.840 & 4.6764 & 13.989 \\
0.85 & 18.628 & 63.288 & 6.9817 & 25.990 \\
0.9 & 34.633 & 158.94 & 12.462 & 60.965 \\
0.95 & 99.14 & 708.8 & 34.31 & 253.7 \\
\hline
\end{tabular}

Aside from providing accurate answers to some very practical questions, the results given in Table 3 are important in that they follow routinely from the technique presented in this paper, are not available in the literature for very deep cracks $(b>0.8 h)$, and are extremely difficult to obtain by using other methods. For example, the solution of the corresponding singular integral equation having a Cauchy-type dominant kernel by using 
a Gaussian integration formula requires a much greater computational effort than the technique presented here for the same accuracy and, for $h>0.8 h$, has an extremely slow convergence.

It may be observed that, as in more conventional formulation of mixed boundary value problems, if the unknown function in (41) is a "flux"-type quantity, then the dominant kernel $k_{s}$ would have a Cauchy-type singularity. In this case too, after properly identifying the fundamental function $w(t)$ (see (42)) of the integral equation that contains the singular behavior of the solution, the problem may again be solved by using the results developed in this paper for Cauchy kernels and by following a procedure similar to that outlined in Sec. 3.

Appendix A: Derivation of formulas (21) and (23). Referring to, for example, [12] the singular integral involving the Jacobi polynomial $P_{n}^{(\alpha, \beta)}$ and its weight may be expressed as

$$
\begin{array}{r}
f_{-1}^{1} \frac{(1-t)^{\alpha}(1+t)^{\beta} P_{n}^{(\alpha, \beta)}(t)}{t-x} d t=\pi \cot \alpha \pi(1-x)^{\alpha}(1+x)^{\beta} P_{n}^{(\alpha, \beta)}(x) \\
-\frac{2^{\alpha+\beta} \Gamma(\alpha) \Gamma(n+\beta+1)}{\Gamma(n+\alpha+\beta+1)} F\left(n+1,-n-\alpha-\beta ; 1-\alpha ; \frac{1-x}{2}\right) \\
\quad(\alpha>-1, \beta>-1, \alpha \neq 0,1,2, \ldots), \quad(\mathrm{A})
\end{array}
$$

where $F(a, b ; c ; z)$ is the hypergeometric and $\Gamma(\alpha)$ is the gamma function. Letting $n=0$, $\alpha=\frac{1}{2}, \beta=0$, and using the properties of hypergeometric functions [13], it can be shown that

$$
f_{-1}^{1} \frac{\sqrt{1-t}}{t-x} d t=-2 \sqrt{2} F\left(1,-\frac{1}{2} ; \frac{1}{2} ; \frac{1-x}{2}\right)=\sqrt{1-x} \log |B|-2 \sqrt{2} \quad(x<1),
$$

where

$$
B=\left(1+\sqrt{\frac{1-x}{2}}\right) /\left(1-\sqrt{\frac{1-x}{2}}\right) .
$$

Following a similar procedure we obtain

$$
f_{-1}^{1} \frac{d t}{(t-x) \sqrt{1-t}}=\sqrt{2} F\left(1, \frac{1}{2} ; \frac{3}{2} ; \frac{1-x}{2}\right)=\frac{\log |B|}{\sqrt{1-x}} \quad(x<1) .
$$

Appendix B: Derivation of formulas (34)-(39). Equations (34)-(39) are obtained by using the expansion

$$
t^{n}=\sum_{i=0}^{n}\left(\begin{array}{c}
n \\
i
\end{array}\right)(t-x)^{i} x^{n-i}
$$


and by referring to the relevant formulas given in Sec. 2 of the paper. For example, from (B1) and (38) it may be shown that

$$
\begin{aligned}
& R_{n}^{j}(x)=f_{-1}^{1} \frac{t^{n} \sqrt{1-t}}{(t-x)^{j}} \\
& =\sum_{i=0}^{j-1}\left(\begin{array}{c}
n \\
i
\end{array}\right) x^{n-i} f_{-1}^{1} \frac{\sqrt{1-t}}{(t-x)^{j-i}} d t+\sum_{i=j}^{n}\left(\begin{array}{c}
n \\
i
\end{array}\right) x^{n-i} \int_{-1}^{1}(t-x)^{i-j} \sqrt{1-t} d t \\
& =\sum_{m=1}^{j}\left(\begin{array}{c}
n \\
j-m
\end{array}\right) x^{n-j+m} f_{-1}^{1} \frac{\sqrt{1-t}}{(t-x)^{m}} d t+\sum_{m=0}^{n-j}\left(\begin{array}{c}
n \\
j+m
\end{array}\right) x^{n-j-m} \int_{-1}^{1}(t-x)^{m} \sqrt{1-t} d t .
\end{aligned}
$$

If we make use of the expression [14]

$$
\int_{-1}^{1}(t-x)^{m} \sqrt{1-t} d t=4 \sqrt{2} \sum_{k=0}^{m}\left(\begin{array}{c}
m \\
k
\end{array}\right) x^{m-k}(-1)^{m} \sum_{p=0}^{k} \frac{(-1)^{p}\left(\begin{array}{l}
k \\
p
\end{array}\right) 2^{k-p}}{2 k-2 p+3}
$$

from (B2) it follows that

$$
\begin{gathered}
R_{n}^{j}(x)=\sum_{m=1}^{j}\left(\begin{array}{c}
n \\
j-m
\end{array}\right) x^{n-j+m} f_{-1}^{1} \frac{\sqrt{1-t}}{(t-x)^{m}} d t+\sum_{k=0}^{n-j} A_{k}^{j} x^{n-j-k} \quad(x<1, n \geqslant 0), \\
A_{k}^{j}=4 \sqrt{2}\left(\begin{array}{c}
n-k-1 \\
j-1
\end{array}\right) \sum_{i=j}^{k}(-1)^{i}\left(\begin{array}{c}
k \\
i
\end{array}\right) \frac{(2)^{i}}{2 i+3} .
\end{gathered}
$$

A similar procedure may be used to derive the formulas given by (34)-(37) and (39), and the related coefficients may be obtained as follows:

$$
\begin{aligned}
& b_{k}= \begin{cases}0 & \text { for } n-k=\text { even } \\
\frac{1}{2 \sqrt{\pi}} \frac{\Gamma\left(\frac{n-k}{2}\right)}{\Gamma\left(\frac{n-k+3}{2}\right)}, & \text { for } n-k=\text { odd },(n \geqslant 0, k \leqslant n+1),\end{cases} \\
& c_{k}= \begin{cases}0, & \text { for } n-k=\text { odd } \\
\frac{(k+1)}{2 \sqrt{\pi}} \frac{\Gamma\left(\frac{n-k-1}{2}\right)}{\Gamma\left(\frac{n-k+2}{2}\right)}, & \text { for } n-k=\text { even }(n \geqslant 0, k \leqslant n),\end{cases} \\
& d_{k}= \begin{cases}0, & \text { for } n-k=\text { even } \\
\frac{1}{\sqrt{\pi}} \frac{\Gamma\left(\frac{n-k}{2}\right)}{\Gamma\left(\frac{n-k+1}{2}\right)}, & \text { for } n-k=\text { odd }(n \geqslant 1, k \leqslant n-1),\end{cases}
\end{aligned}
$$




$$
\begin{aligned}
& e_{k}= \begin{cases}0, & \text { for } n-k=\text { odd } \\
\frac{(k+1)}{\sqrt{\pi}} \frac{\Gamma\left(\frac{n-k-1}{2}\right)}{\Gamma\left(\frac{n-k}{2}\right)}, & \text { for } n-k=\text { even }(n \geqslant 2, k \leqslant n-2),\end{cases} \\
& B_{k}^{j}=2 \sqrt{2}\left(\begin{array}{c}
n-k-1 \\
j-1
\end{array}\right) \sum_{i=0}^{k}(-1)^{i}\left(\begin{array}{c}
k \\
i
\end{array}\right) \frac{(2)^{i}}{2 i+1} \quad(n \geqslant 0),
\end{aligned}
$$

where for the gamma function we have $\Gamma\left(-\frac{1}{2}\right)=-2 \Gamma\left(\frac{1}{2}\right)=-2 \sqrt{\pi}$.

Some examples of the polynomials $B_{n}, C_{n}, D_{n}$, and $E_{n}$ and some useful recursion formulas are given below.

$$
\begin{aligned}
& B_{0}=-x, \quad C_{0}=-1, \\
& B_{1}=-x^{2}+\frac{1}{2}, \quad C_{1}=-2 x, \\
& B_{2}=-x^{3}+\frac{x}{2}, \quad C_{2}=-3 x^{2}+\frac{1}{2}, \\
& B_{3}=-x^{4}+\frac{x^{2}}{2}+\frac{1}{8}, \quad C_{3}=-4 x^{3}+x, \\
& B_{4}=-x^{5}+\frac{x^{3}}{2}+\frac{x}{8}, \quad C_{4}=-5 x^{4}+\frac{3}{2} x^{2}+\frac{1}{8}, \\
& B_{5}=-x^{6}+\frac{x^{4}}{2}+\frac{x^{2}}{8}+\frac{1}{16}, \quad C_{5}=-6 x^{5}+2 x^{3}+\frac{x}{4}, \\
& D_{0}=0, \quad E_{0}=0, \\
& D_{1}=1, \quad E_{1}=0, \\
& D_{2}=x, \quad E_{2}=1, \\
& D_{3}=x^{2}+\frac{1}{2}, \quad E_{3}=2 x, \\
& D_{4}=x^{3}+\frac{x}{2}, \quad E_{4}=3 x^{2}+\frac{1}{2} \text {, } \\
& D_{5}=x^{4}+\frac{x^{2}}{2}+\frac{3}{8}, \quad E_{5}=4 x^{3}+x, \\
& C_{n+1}(x)-x C_{n}(x)=B_{n}(x), \\
& E_{n+1}(x)-x E_{n}(x)=D_{n}(x) \text {, } \\
& D_{n}(x)-D_{n+2}(x)=B_{n}(x) \text {, } \\
& E_{n}(x)-E_{n+2}(x)=C_{n}(x) \text {, } \\
& R_{n+1}^{j+1}(x)-x R_{n}^{j+1}(x)=R_{n}^{j}(x), \\
& S_{n+1}^{j+1}(x)-x S_{n}^{j+1}(x)=S_{n}^{j}(x),
\end{aligned}
$$




$$
\begin{aligned}
S_{n}^{j}(x)-S_{n+1}^{j}(x) & =R_{n}^{j}(x), \\
\frac{\partial}{\partial x} R_{n}^{j}(x) & =j R_{n}^{j+1}(x), \\
\frac{\partial}{\partial x} S_{n}^{j}(x) & =j S_{n}^{j}(x) .
\end{aligned}
$$

Appendix C: The elasticity problem considered as an example. The two-dimensional elasticity problem considered in Sec. 4 and described in Fig. 1 requires the solution of differential equations

$$
\nabla^{2} u_{i}+\frac{2}{\kappa-1} u_{k, k i}=0 \quad(i, k=x, y),
$$

subject to

$$
\begin{gathered}
\sigma_{x x}(0, y)=\sigma_{x y}(0, y)=\sigma_{x x}(h, y)=\sigma_{x y}(h, y)=0 \quad(-\infty<y<\infty), \\
\sigma_{x y}(x, 0)=0 \quad(0<x<h), \\
\left.\begin{array}{c}
\sigma_{y y}(x, 0)=-p(x) \quad(a<x<b), \\
u_{y}(x, 0)=0 \quad(0<x<a, b<x<h),
\end{array}\right\}
\end{gathered}
$$

where $u_{x}, u_{y}$ are the $x, y$ components of the displacement vector, $\sigma_{i j}$ is the stress tensor $(i, j=x, y)$, and $\kappa$ is an elastic constant $(\kappa=3-4 \nu$ for plane strain and $\kappa=(3-$ $\nu) /(1+\nu)$ for plane stress, $\nu$ being the Poisson's ratio). The stress and displacement components are related through

$$
\begin{aligned}
\sigma_{x x} & =\frac{\mu}{\kappa-1}\left[(1+\kappa) u_{x, x}+(3-\kappa) u_{y, y}\right], \\
\sigma_{y y} & =\frac{\mu}{\kappa-1}\left[(1+\kappa) u_{y, y}+(3-\kappa) u_{x, x}\right], \\
\sigma_{x y} & =\mu\left(u_{x, y}+u_{y, x}\right) .
\end{aligned}
$$

The solution of (C1) satisfying the symmetry condition (C3) may be expressed as [10]

$$
\begin{aligned}
u_{i}(x, y) & =u_{i}^{c}+u_{i}^{1}+u_{1}^{2}, \quad(i=x, y), \\
u_{x}^{c}(x, y) & =\frac{1}{2 \pi(1+\kappa)} \int_{a}^{b} V(t)\left[-(\kappa-1) \frac{x-t}{r^{2}}+\frac{4(x-t) y^{2}}{r^{4}}\right] d t, \\
u_{y}^{c}(x, y) & =\frac{1}{2 \pi(1+\kappa)} \int_{a}^{b} V(t)\left[(\kappa-1) \frac{y}{r^{2}}+\frac{4 y^{3}}{r^{4}}\right] d t, \\
r^{2} & =(x-t)^{2}+y^{2}, \\
u_{x}^{1}(x, y) & =\frac{2}{\pi} \int_{0}^{\infty}\left[A_{1}+\left(\frac{\kappa}{\alpha}+x\right) A_{2}\right] e^{-\alpha x} \cos \alpha y d \alpha, \\
u_{y}^{1}(x, y) & =\frac{2}{\pi} \int_{0}^{\infty}\left(A_{1}+A_{2} x\right) e^{-\alpha x} \sin \alpha y d \alpha,
\end{aligned}
$$




$$
\begin{aligned}
& u_{x}^{2}(x, y)=-\frac{2}{\pi} \int_{0}^{\infty}\left[B_{1}+\left(\frac{\kappa}{\beta}+h-x\right) B_{2}\right] e^{-\beta(h-x)} \cos \beta y d \beta, \\
& u_{y}^{2}(x, y)=\frac{2}{\pi} \int_{0}^{\infty}\left[B_{1}+B_{2}(h-x)\right] e^{-\beta(h-x)} \sin \beta y d \beta,
\end{aligned}
$$

where $V$ is the auxiliary function defined by

$$
V(x)=u_{y}(x,+0)-u_{y}(x,-0) \quad(a<x<b) .
$$

In this solution $u_{i}^{c}, u_{i}^{1}$, and $u_{i}^{2}$ are respectively associated with an infinite plane having a crack and the half-planes $x>0$ and $x<h$. The corresponding expressions for the stress components are given as follows:

$$
\begin{aligned}
& \frac{1}{2 \mu} \sigma_{x x}^{c}=\frac{1}{\pi(1+\kappa)} \int_{a}^{b} V(t)\left\{\frac{1}{r^{2}}-\frac{8(x-t)^{2} y^{2}}{r^{6}}\right\} d t, \\
& \frac{1}{2 \mu} \sigma_{y y}^{c}=\frac{1}{\pi(1+\kappa)} \int_{a}^{b} V(t)\left\{\frac{1}{r^{2}}-\frac{4 y^{2}}{r^{4}}+\frac{8(x-t)^{2} y^{2}}{r^{6}}\right\} d t, \\
& \frac{1}{2 \mu} \sigma_{x y}^{c}=\frac{1}{\pi(1+\kappa)} \int_{a}^{b} V(t)\left\{\frac{2(x-t) y}{r^{4}}-\frac{8(x-t) y^{3}}{r^{6}}\right\} d t, \\
& \frac{1}{2 \mu} \sigma_{x x}^{1}=-\frac{2}{\pi} \int_{0}^{\infty}\left[\alpha\left(A_{1}+A_{2} x\right)+\left(\frac{\kappa+1}{2}\right) A_{2}\right] e^{-\alpha x} \cos \alpha y d \alpha, \\
& \frac{1}{2 \mu} \sigma_{y y}^{1}=\frac{2}{\pi} \int_{0}^{\infty}\left[\alpha\left(A_{1}+A_{2} x\right)+\left(\frac{\kappa-3}{2}\right) A_{2}\right] e^{-\alpha x} \cos \alpha y d \alpha, \\
& \frac{1}{2 \mu} \sigma_{x y}^{1}=-\frac{2}{\pi} \int_{0}^{\infty}\left[\alpha\left(A_{1}+A_{2} x\right)+\left(\frac{\kappa-1}{2}\right) A_{2}\right] e^{-\alpha x} \sin \alpha y d \alpha, \\
& \frac{1}{2 \mu} \sigma_{x x}^{2}=-\frac{2}{\pi} \int_{0}^{\infty}\left[\beta\left(B_{1}+B_{2}(h-x)\right)+\left(\frac{\kappa+1}{2}\right) B_{2}\right] e^{-\beta(h-x)} \cos \beta y d \beta, \\
& \frac{1}{2 \mu} \sigma_{y y}^{2}=\frac{2}{\pi} \int_{0}^{\infty}\left[\beta\left(B_{1}+B_{2}(h-x)\right)+\left(\frac{\kappa-3}{2}\right) B_{2}\right] e^{-\beta(h-x)} \cos \beta y d \beta, \\
& \frac{1}{2 \mu} \sigma_{x y}^{2}=\frac{2}{\pi} \int_{0}^{\infty}\left[\beta\left(B_{1}+B_{2}(h-x)\right)+\left(\frac{\kappa-1}{2}\right) B_{2}\right] e^{-\beta(h-x)} \sin \beta y d \beta .
\end{aligned}
$$

Using the homogeneous boundary conditions (C2), the unknown functions $A_{1}(\alpha), A_{2}(\alpha)$, $B_{1}(\beta)$, and $B_{2}(\beta)$ can be expressed in terms of $V(x)$ and the mixed boundary conditions (C4) may then be shown to reduce to the integral Eq. (50) given in Sec. 4.

Appendix D: Stress intensity factor for the edge crack calculated from Koiter's results. The edge crack problem in a semi-infinite plane has been considered in the literature many times and mostly for comparison. The stress intensity factor 1.1215 has become a standard when comparing numerical techniques for the solution of singular integral equations. For uniform pressure $p_{0}$ applied on the crack surface, a closed-form expression for the stress intensity factor in terms of an infinite integral is given by Koiter [11]:

$$
\frac{k_{1}}{p_{0} \sqrt{b}}=\frac{\sqrt{2(B+1)}}{\sqrt{\pi} A},
$$


where $A$ is calculated from

$$
\log A=-\frac{1}{\pi} \int_{0}^{\infty} \frac{1}{1+\alpha^{2}} \log \left(\frac{\alpha \sinh \pi \alpha}{\sqrt{B^{2}+\alpha^{2}}\left[\cosh \pi \alpha-2 \alpha^{2}-1\right]}\right) d \alpha
$$

and $B$ is an arbitrary constant greater than 1 .

The result is independent of the choice for $B$ and numerical calculations show that

$$
\frac{k_{1}}{p_{0} \sqrt{b}} \cong 1.12152226
$$

where there may be an error only in the last digit.

Acknowledgments. The research reported in this paper was supported by NSF under grant MEA-8209083 and by NASA-Langley under grant NGR 39-007-011 and was completed when the second author was an Alexander von Humboldt Senior U. S. Scientist Awardee in Freiburg (i.Br.), Germany.

\section{REFERENCES}

[1] J. Hadamard, Lectures on Cauchy's problem in linear partial differential equations, Yale University Press, 1923.

[2] L. Schwartz, Théorie des distributions, Vols. I and II, Hermann, 1957-1959

[3] K. W. Mangler, Improper integrals in theoretical aerodynamics, Royal Aircraft Establishment, Farnborough, Report No. 2424 (1951)

[4] H. R. Kutt, The numerical evaluation of principal value integrals by finite-part integration, Numer. Math. 24, 205-210 (1975)

[5] H. R. Kutt, Quadrature formulae for finite-part integrals, Special Report WISK 178, Pretoria, National Research Institute for Mathematical Sciences, 1975

[6] H. R. Kutt, On the numerical evaluation of finite-part integrals involving an algebraic singularity, Special Report WISK 179, Pretoria, National Research Institute for Mathematical Sciences, 1975

[7] N. I. Muskhelishvili, Singular integral equations, Noordhoff, Groningen, 1953

[8] N. I. Ioakimidis, On the numerical evaluation of derivatives of Cauchy principal value integrals, Computing 27. $81-88(1981)$

[9] C. T. H. Baker, The numerical treatment of integral equations, Clarendon Press, Oxford (1977)

[10] A. C. Kaya, Applications of integral equations with strong singularities in fracture mechanics, Ph. D. thesis, Lehigh University, 1984

[11] W. T. Koiter, Discussion of "Rectangular tensile sheet with symmetrical edge cracks," by O. L. Bowie, J. Appl. Mech. 32, Trans. ASME 87, Series E, 237 (1965)

[12] F. G. Tricomi, On the finite Hilbert transformation, Quart. J. Math. Oxford Ser. (2) 2, 199-211 (1951)

[13] M. Abramowitz and I. A. Stegun, ed., Handbook of mathematical functions, Dover Publications, Inc., New York, 1965.

[14] I. S. Gradshtein and I. M. Ryzhik, Table of integrals, series and products, Academic Press, New York, 1965 